\title{
Low-frequency noise and thermal equilibrium properties of vacancies
}

Sofie Beyne, ${ }^{1,2, a)}$ Ingrid De Wolf, ${ }^{1,2}{ }^{2}$ zsolt Tökei, ${ }^{2}$ and Kristof Croes $^{2}$

1) MTM, KU Leuven, Kasteelpark Arenberg 44 bus 2450, B-3001, Leuven, Belgium

${ }^{2)}$ imec, Kapeldreef 75, B-3001, Leuven, Belgium

(Dated: 12 February 2020)

A model explaining Lorentzian low-frequency (LFN) noise spectra observed in electronic interconnects is presented. The model is based on the interaction of electrons with vacancies, whose thermodynamic equilibrium properties determine the temperature dependence of the noise. The model therefore allows calculating vacancy formation and migration enthalpies. It is experimentally verified on copper and tungsten interconnects; the sum of formation and migration enthalpies matches the electromigration (EM) activation energy obtained using standard accelerated tests.

Previously, a new electromigration (EM) test method, based on low-frequency noise (LFN) measurements was proposed $^{1-3}$. Contrary to standard test methods, LFN provides non-destructive and faster EM characterization, while enabling a deeper fundamental understanding of EM mechanisms ${ }^{1-5}$. A particularly interesting application of the LFN measurements is the calculation of EM activation energies, based on the model of Dutta et al. ${ }^{6,7}$. Recently, we showed an adaptation of that model, in order to explain why the LFN activation energy correlates so well with the EM activation energy in electronic interconnects ${ }^{3}$. Nevertheless, it could still not be used to calculate thermodynamic equilibrium properties of vacancies. In this letter, we provide an alternative explanation to incorporate the calculation of vacancy formation and migration enthalpies from LFN measurements. The main benefit of this technique is the individual calculation of the thermodynamic equilibrium properties of defects. This is very helpful to obtain a better physical understanding of the diffusion mechanisms acting in a metal, for example during electromigration failure. The activation energy derived using standard EM tests (black's law) can sometimes be an average of different mechanisms and extrapolation to use conditions may not always be justified. Obtaining vacancy migration and formation enthalpies at operation conditions can fill the gaps of standard EM testing and provide additional fundamental understanding. Two interconnect systems can have a similar activation energy but different vacancy formation and migration enthalpies. Identifying those dif ferences can help to understand the impact of different process parameters on electromigration. For example, a low vacancy formation enthalpy could indicate preexisting defects. In summary, this methodology allows comparing different interconnect material systems in detail, with the additional benefit of being non-destructive. The model presented here, is similar in approach to that of Stoll et al. ${ }^{8}$; it is assumed that the continuous creation and annihilation of vacancies in the metal and the interaction of electrons therewith, result in pulses in the output voltage. The final signal is a superposition of sev-

a) sofie.beyne@imec.be eral of these pulses, as shown in Fig. 1. The pulse height

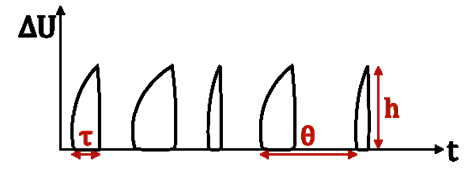

FIG. 1. Schematic representation of voltage pulses caused by a vacancy. The shape $y(t)$ is defined by the height $h$ of the pulse and the lifetime of the vacancy, $\tau$.

is given by $h$, the distance between two adjacent pulses is $\theta$ and the duration of the pulse, which is the lifetime of the vacancy, is described by $\tau$. The shape of a pulse is described by $y(t)$. To calculate the corresponding power spectrum, it suffices to first calculate the spectrum for the individual pulses shown in Fig. $1^{9}$.

The following assumptions are made ${ }^{9}$ : (a) The pulse sequences are stationary and ergodic (the time average is equal to the average over the probability space), (b) independent parameters of different pulses and (c) all pulses have the same basic shape $y(t)$.

Heiden showed that in the case of independent $\theta, \tau$ and $h$, with $\langle h\rangle=0$, the power spectrum is given by ${ }^{9}$ :

$$
S(f)=2 \nu\left\langle h^{2}\right\rangle\left\langle\left|F_{0}(f, \tau)\right|^{2}\right\rangle,
$$

with $\nu$ the pulse density and $\left\langle\left|F_{0}(f, \tau)\right|^{2}\right\rangle$ the Fourier transformation of an individual pulse. Eq. 1 is valid irrespective of the distribution of $\tau$ and $\theta$, but only when the duration and amplitude of the pulse are not coupled. For a single pulse series, the number of pulses in a time $\Delta t$ is equal to $\Delta t /\langle\tau\rangle$, where $\langle\tau\rangle$ is the average vacancy lifetime. The pulse density $\nu$ is the average number of pulses per unit time and is therefore given by $\langle\tau\rangle^{-1}$.

Because several of these pulse series occur simultaneously, albeit independently, the spectrum in eq. (1) can be multiplied by $N_{V}$, the average number of vacancies. The total vacancy pulse density is then given by

$$
\nu_{t o t}=\frac{N_{V}}{\langle\tau\rangle} .
$$

If the creation and annihilation of a vacancy is considered to be statistically independent, with each vacancy having 
a lifetime $\tau$, an exponential distribution of the lifetimes may be assumed ${ }^{8,10}$. The probability distribution function is then given by:

$$
P(\tau)=\frac{1}{\tau_{0}} \exp \left(-\frac{\tau}{\tau_{0}}\right),
$$

where $1 / \tau_{0}$ is the rate parameter and $\tau_{0}$ is the mean and standard deviation of the distribution. Moreover, for a rectangular pulse:

$$
F_{0}(f, \tau)=\int_{0}^{\tau} \exp (-j 2 \pi f t) d t
$$

such that with $\left\langle h^{2}\right\rangle=(\Delta U)^{2}$, eq. (1) becomes:

$$
S(f)=\frac{4 N_{V}}{(2 \pi f)^{2} \tau_{0}}(\Delta U)^{2}\left[1-\int_{0}^{\infty} P(\tau) \cos (2 \pi f \tau) d \tau\right] .
$$

After substituting expression (3) and calculation of the integral, the spectrum is then:

$$
S(f)=4 N_{V}(\Delta U)^{2} \frac{\tau_{0}}{(2 \pi f)^{2} \tau_{0}^{2}+1} .
$$

This same result was obtained by Stoll ${ }^{8}$ using both the fluctuation dissipation theorem and by considering the power spectrum of a series of single pulses, similar to the analysis presented above.

The expression for the PSD in eq. 6 contains the following vacancy properties: $N_{V}$, the number of vacancies in thermal equilibrium and $\tau_{0}=\langle\tau\rangle$, the average vacancy lifetime. Furthermore

$$
N_{V}=C_{V} C_{A} V
$$

where $C_{A}$ is the atom concentration, $V$ the volume of the sample under investigation and $C_{V}$ the vacancy concentration at a temperature $\mathrm{T}^{11}$, given by:

$$
C_{V}=\exp \left(-\frac{G_{V}^{F}}{k T}\right)=\exp \left(-\frac{H_{V}^{F}-T S_{V}^{F}}{k T}\right) .
$$

$H_{V}^{F}$ and $S_{V}^{F}$ are the enthalpy and entropy of vacancy formation respectively, $k$ is the Boltzmann constant. $G_{V}^{F}$ and $G_{V}^{M}$ are the corresponding Gibbs free energies of vacancy formation and migration, defined as $G=H-T S$. The average lifetime of a vacancy can be calculated from the average distance it can travel by a random walk in the lattice, until it is annihilated. During an average lifetime $\langle\tau\rangle$, the vacancy makes $N_{S}$ steps of length equal to the lattice parameter $a$. The total distance which the vacancy travels, is the result of a random walk and is given by

$$
\Lambda=a \sqrt{N_{S}},
$$

while the total number of steps depends on the jump frequency, $\Gamma$, and the lifetime:

$$
N_{S}=\langle\tau\rangle \cdot \Gamma .
$$

This vacancy jump frequency is given by ${ }^{11}$ :

$$
\Gamma=C_{M} \frac{\bar{v}}{\delta},
$$

where $C_{M}$ is the concentration of activated complexes (atoms in a saddle point configuration) that have to overcome an energy barrier $G_{V}^{M}$ to migrate. $\bar{v}$ is the velocity of the atoms moving through the midpoint with width $\delta$ such that $\frac{\bar{v}}{\delta}$ is equal to the Debye frequency $\nu_{0}$. The jump frequency $\Gamma$ is thus also given by:

$$
\Gamma=\nu_{0} \exp \left(-\frac{G_{V}^{M}}{k T}\right)=\nu_{0} \exp \left(-\frac{H_{V}^{M}-T S_{V}^{M}}{k T}\right) .
$$

The vacancy lifetime $\langle\tau\rangle=\tau_{0}$ can then be calculated by combining equations (9) and (10):

$$
\langle\tau\rangle=\frac{\Lambda^{2}}{a^{2} \Gamma},
$$

such that the spectrum, given by eq. (6) becomes:

$$
\begin{aligned}
S(f) & =4 \frac{\Lambda^{2}}{a^{2}} \nu_{0}^{-1}(\Delta U)^{2} C_{A} V \exp \left(\frac{S_{V}^{F}-S_{V}^{M}}{k}\right) \\
\cdot & \frac{\exp \left(-\frac{H_{V}^{F}-H_{V}^{M}}{k T}\right)}{(2 \pi f)^{2} \frac{\Lambda^{4}}{a^{4}} \nu_{0}^{-2} \exp \left(\frac{2 H_{V}^{M}}{k T}\right) \exp \left(\frac{-2 S_{V}^{M}}{k}\right)+1} .
\end{aligned}
$$

This result is valid for different types of vacancy diffusion, for example grain boundary, interface or surface diffusion (common in electromigration of microelectronic interconnects). The 3 assumptions made in the first paragraph are independent of the type of vacancy diffusion. The activation energies however, will differ because the thermodynamic equilibrium properties $\left(G_{V}^{M}, G_{V}^{F}\right)$ for each of these diffusion mechanisms are different.

The spectrum in eq. (14) is of Lorentzian form and is illustrated in Fig. 2. Two regions can be distinguished: at low frequencies (1), the PSD is frequency-independent and at higher frequencies (2), the PSD decreases as $1 / f^{2}$. The frequency at which the transition from (1) to (2) occurs, is denoted by $f_{\text {knee }}$ :

$$
f_{\text {knee }} \propto \exp \left(-\frac{H_{V}^{M}}{k T}\right),
$$

The two regions will now be discussed in detail.

The frequency independent part occurs for $f \rightarrow 0$. In this case, eq. (14) reduces to

$$
\begin{aligned}
& \lim _{f \rightarrow 0} S(f)=4 \frac{\Lambda^{2}}{a^{2}} \nu_{0}^{-1}(\Delta U)^{2} C_{A} V \\
& \cdot \exp \left(\frac{S_{V}^{F}-S_{V}^{M}}{k}\right) \exp \left(-\frac{H_{V}^{F}-H_{V}^{M}}{k T}\right) .
\end{aligned}
$$

From the temperature dependence of $\lim _{f \rightarrow 0} S(f)$, we can then calculate $\left(H_{V}^{F}-H_{V}^{M}\right)$, because:

$$
\ln (S(f \rightarrow 0))=-\frac{\left(H_{V}^{F}-H_{V}^{M}\right)}{k T}+C .
$$




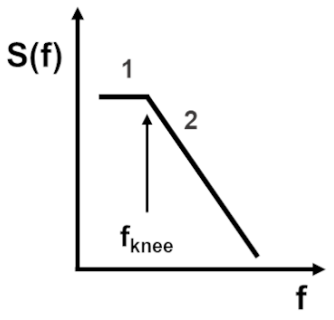

FIG. 2. Illustration of the Lorentzian spectrum given by eq. (14). Two parts can be distinguished: (1) a frequencyindependent part at low frequencies and (2) a $1 / f^{2}$ dependency at higher frequencies.

Above the frequency $f_{k n e e}$ the power spectrum becomes frequency dependent. Considering that for sufficiently large $x: \ln (1+x) \propto \ln (x)$, this means that if

$$
(2 \pi f)^{2} \frac{\Lambda^{4}}{a^{4}} \nu_{0}^{-2} \exp \left(\frac{2 H_{V}^{M}-2 T S_{V}^{M}}{k T}\right)>0,
$$

while being sufficiently large (which depends not only on the thermodynamic properties of the vacancies, but also on the ratio $\left.\frac{\Lambda^{4}}{a^{4}}\right)$, the following approximation can be made:

$$
\ln (S(f)) \propto-\frac{\left(H_{V}^{F}+H_{V}^{M}\right)}{k T}+C^{\prime} .
$$

The slope of $\ln (S(f))$ over $1 / k T$ (evaluated at frequencies in the two regions of the spectrum), is then proportional to either $\left(H_{V}^{F}-H_{V}^{M}\right)$ or $\left(H_{V}^{F}+H_{V}^{M}\right)$, such that the vacancy formation and migration enthalpies can be calculated individually. $H_{V}^{M}$ could also be obtained directly from eq. (15), but it is generally difficult to correctly extract $f_{k n e e}$ from the experimental data.

$H_{V}^{F}$ and $H_{V}^{M}$, are directly linked with electromigration because also there, mass-transport occurs by vacancyassisted diffusion. The vacancy diffusion coefficient in an FCC lattice, such as for $\mathrm{Cu}$, can be calculated as ${ }^{11}$ :

$$
D=a^{2} C_{V} \Gamma
$$

where $a$ is again the inter-atomic distance, $C_{V}$ the fraction of vacant sites, as in eq. (8) and $\Gamma$, the jump frequency as in eq. (12), such that

$$
D=a^{2} \nu_{0} \exp \left(-\frac{G_{V}^{F}+G_{V}^{M}}{k T}\right)=a^{2} \nu_{0} \exp \left(-\frac{E_{A}}{k T}\right) .
$$

Because the term $\exp \left(\frac{S_{V}^{F}+S_{V}^{M}}{k}\right)$ is generally negligible, this $E_{A}$ is indeed equal to the sum of the enthalpy of formation and migration, or as per eq. (19), the slope of $\ln (S(f))$ vs $1 / k T$, when condition (18) is fulfilled.

This theory is now experimentally verified on a $22 \mathrm{~nm}$ wide and $100 \mu \mathrm{m}$ long $\mathrm{Cu}$ interconnect with a $3 \mathrm{~nm}$

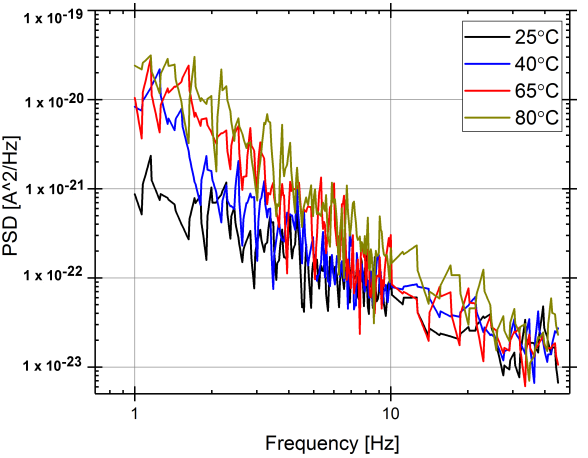

FIG. 3. Power spectral density from 1 to $50 \mathrm{~Hz}$ at $25,40,65$ and $80^{\circ} \mathrm{C}$ of a $22 \mathrm{~nm}$ wide and $100 \mu \mathrm{m}$ long $\mathrm{Cu}$ interconnect with a $3 \mathrm{~nm}$ TaN barrier and $1 \mathrm{~nm}$ Co liner. Other temperatures are omitted for the purpose of readability.

TaN barrier and $1 \mathrm{~nm}$ Co liner. The LFN measurement setup that was used to obtain the spectra at different temperatures can be found in ref. ${ }^{2}$.

The measured spectra at a few temperatures are shown in Fig. 3.

Similar to the illustration of the expected spectrum in Fig. 2, these spectra decrease with the frequency as $\approx 1 / f^{2}$ and exhibit a 'plateau', where the PSD is frequency independent. Above $65^{\circ} \mathrm{C}$, the spectra start to deviate more from the Lorentzian type depicted in Fig. 2. At the temperatures where the PSD is independent between 1 and $2 \mathrm{~Hz}$, eq. (17) can be verified by plotting $\ln (\mathrm{PSD}(1 \mathrm{~Hz}))$ as a function of $1 / k T$ and calculating the slope. This is shown in Fig. 4, where $\left(H_{V}^{F}-H_{V}^{M}\right)$ is then found to be $0.59 \pm 0.08 \mathrm{eV}$. When the PSD is evaluated at $2 \mathrm{~Hz}\left(>f_{\text {knee }}\right)$, its temperature dependence follows eq. (19). In Fig. 4, $\ln (\operatorname{PSD}(2 \mathrm{~Hz}))$ is plotted as a function of $1 / k T$ and the slope is $-0.81 \pm 0.06 \mathrm{eV}$, which is equal to $-\left(H_{V}^{F}+H_{V}^{M}\right)$. Using both results, we then find that in these $\mathrm{Cu}$ interconnects, $H_{V}^{F}=0.7 \pm 0.07 \mathrm{eV}$ and $H_{V}^{M}=0.11 \pm 0.07 \mathrm{eV}$

Note that in Fig. 4, the multiple data points at each temperature are the result of different measurements. Not all LFN measurements were equally reliable, due to external interference with the measurements. Only the reliable LFN data, exhibiting a Lorentzian noise spectrum (to comply with the model's assumptions) were used.

The value of $H_{V}^{M}$ can also be obtained from the temperature dependence of $f_{\text {knee }}$, using eq. (15). Determining the exact value of $f_{k n e e}$ is, however, difficult because of the noisiness of the curves (see Fig. 3). Nevertheless, the calculation was done here and the results are shown in Fig. 5. The slope, which is equal to $-H_{V}^{M}$, is $-0.08 \pm 0.02 \mathrm{eV}$. This is indeed in accordance with the value calculated using the temperature dependence of 


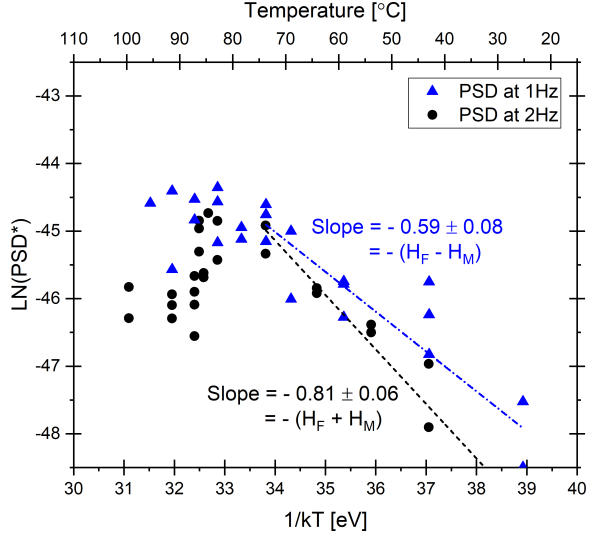

FIG. 4. $\ln (\mathrm{PSD}(1 \mathrm{~Hz}))$ and $\ln (\mathrm{PSD}(2 \mathrm{~Hz}))$ are plotted as a function of $1 / k T$, for a $22 \mathrm{~nm}$ wide and $100 \mu \mathrm{m}$ long $\mathrm{Cu}$ interconnect with a $3 \mathrm{~nm}$ TaN barrier and $1 \mathrm{~nm}$ Co liner. The respective slopes are equal to $-\left(H_{V}^{F}-H_{V}^{M}\right)$ and $-\left(H_{V}^{F}+H_{V}^{M}\right)$.

the PSD magnitude. In Fig. 5, $f_{k n e e}$ in the vicinity of the peak is observed to deviate from the overall trend Nevertheless these points are shown because the $f_{\text {knee }}$ could still be extracted.

The sum of the vacancy formation and migration enthalpies is indeed in line with the electromigration activation energy $(0.85 \pm 0.07 \mathrm{eV})$ and equal to the activation energy, previously obtained using the Dutta model $^{2,12}$

Note that above $70^{\circ} \mathrm{C}$, the spectra deviate from the Lorentzian in Fig. 2. Moreover, around this temperature

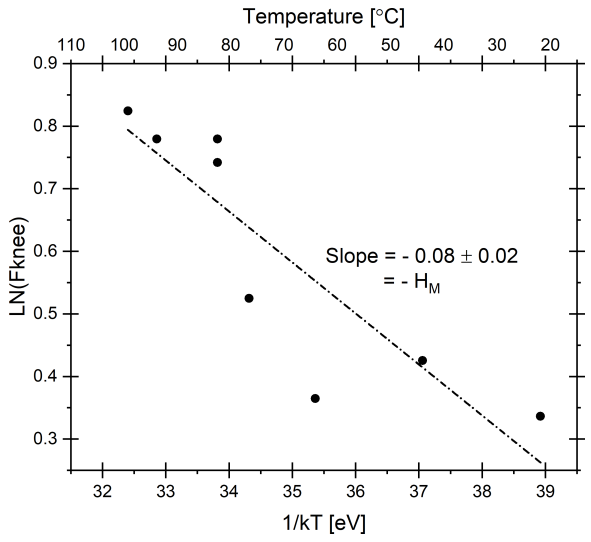

FIG. $5 . \ln \left(f_{\text {knee }}\right)$ vs. $1 / k T, f_{k n e e}$ is the frequency at which the plateau starts.
TABLE I. Summary of $H_{V}^{F}$ and $H_{V}^{M}$ obtained from the LFN measurements for the $\mathrm{Cu}$ and $\mathrm{W}$ lines. The last column shows the EM activation energy obtained using standard accelerated testing (Black's law).

\begin{tabular}{c|c|c|c} 
& $\mathbf{H}_{\mathbf{V}}^{\mathbf{F}}$ & $\mathbf{H}_{\mathbf{V}}^{\mathbf{M}}$ & EM E \\
\hline $\mathbf{C u}$ & $0.7 \pm 0.07 \mathrm{eV}$ & $0.11 \pm 0.07 \mathrm{eV}$ & $0.85 \pm 0.07 \mathrm{eV}$ \\
\hline $\mathbf{W}$ & $0.5 \pm 0.06 \mathrm{eV}$ & $0.14 \pm 0.05 \mathrm{eV}$ & $0.69 \mathrm{eV}$ \\
\hline
\end{tabular}

the LFN PSD exhibits a maximum (can be seen in Fig. 4 ), due to the activation of a diffusion mechanism ${ }^{3,6}$. This maximum then results in a change in slope, such that the above model is no longer applicable. Therefore, the slope is calculated based on the data points at temperatures below those of the PSD maximum.

The model is also tested on tungsten interconnects; the results are shown in Fig. 6. For tungsten, the cut-off frequency in the 25 to $100^{\circ} \mathrm{C}$ range, occurs between 2 and $6 \mathrm{~Hz}$, hence the choice to use the PSD at $2 \mathrm{~Hz}$ to calculate $H_{V}^{F}-H_{V}^{M}$ and the PSD at $10 \mathrm{~Hz}$ to obtain $H_{V}^{F}+H_{V}^{M}$. Using the data from Fig. 6 (a), the vacancy formation enthalpy, $H_{V}^{F}$, is $0.50 \pm 0.06 \mathrm{eV}$ and the vacancy migration enthalpy, $H_{V}^{M}$, is $0.14 \pm 0.05 \mathrm{eV}$. The alternative extraction of $H_{V}^{M}$, from the temperature dependence of $f_{k n e e}$, is demonstrated in Fig.6 (b) and gives $H_{V}^{M}=0.15 \pm 0.03 \mathrm{eV}$, which is perfectly in line with the the value found using Fig. 6 (a). As discussed in our publication $^{1}$, the EM activation energy $\left(H_{V}^{F}+H_{V}^{M}\right)$ was found to be $0.69 \mathrm{eV}$ using standard accelerated EM tests and $0.73-0.75 \mathrm{eV}$ using LFN with the model of Dutta et al.

Note that also in this case the PSD at $2 \mathrm{~Hz}$ shows a maximum in PSD close to $65^{\circ} \mathrm{C}$. Also in this case, that can be explained by application of the model of Dutta et al. ${ }^{6}$, but is out of the scope of this paper.

The results from both experiments are summarized in Table I, where the last column shows the electromigration activation energy obtained using standard accelerated testing. For both $\mathrm{W}$ and $\mathrm{Cu}, H_{V}^{F}+H_{V}^{M}$, is in line with the $\mathrm{EM} \mathrm{E}_{\mathrm{A}}$. The vacancy equilibrium properties in the $\mathrm{Cu}$ and $\mathrm{W}$ interconnects are very similar, which is not surprising given their similar EM activation energy. The low tungsten $\mathrm{E}_{\mathrm{A}}$ was previously discussed in ref. ${ }^{1}$. It should be noted that these $W$ samples had significant voids in the center of the line, which could contribute to their low vacancy formation enthalpy.

In conclusion, this letter presents a model that attributes the LFN spectra observed in metallic interconnects, to the interaction of electrons with vacancies. The temperature dependence of the LFN PSD can then be explained by the thermodynamic equilibrium properties of vacancies. The model allows extracting the enthalpies of vacancy formation and migration by analyzing the temperature dependence of the LFN in two different regimes and was verified experimentally.

As compared to the model of Dutta et al. and adapta- 
(a)

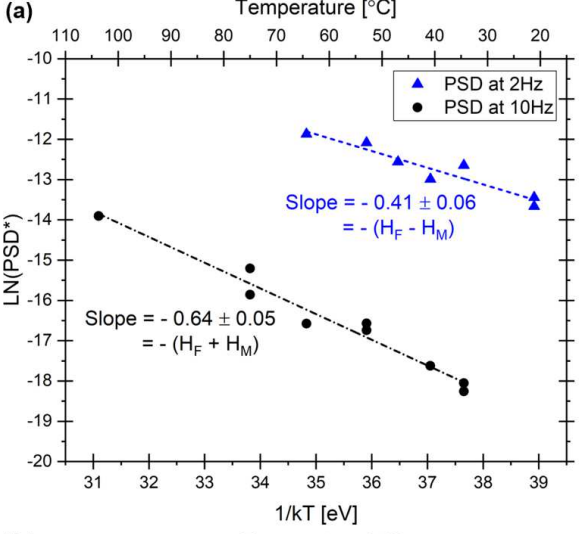

(b)

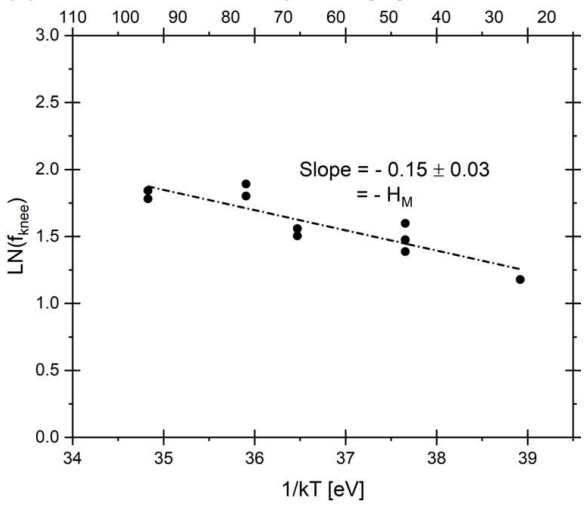

FIG. 6. The data in this figure were obtained on $32 \mathrm{~nm}$ wide and $100 \mu \mathrm{m}$ long tungsten interconnects; (a) shows the logarithm of the PSD at 2 and $10 \mathrm{~Hz}$ as a function of $1 / k T$, with the respective slopes equal to $H_{V}^{F}-H_{V}^{M}$ and $H_{V}^{F}+H_{V}^{M}$. (b) displays the logarithm of the cut-off frequency $\left(f_{k n e e}\right)$ as a function of $1 / k T$, the slope being equal to $H_{V}^{M}$.

tions thereof, the disadvantages of this model are that it is only valid in the case of a $1 / f^{2}$ spectrum, when one mechanism is dominant and that the calculation of the activation energy, based on a slope, introduces a large uncertainty. Nevertheless, the insights that this model offers into the equilibrium properties of vacancies enables many applications. For example, the comparison of vacancy properties in different materials and providing a means for fast and non-destructive EM characterization at a much more fundamental level than is possible with the standard accelerated EM test methods. In future work, the model presented in this paper could be expanded to include more complex vacancy diffusion scenarios, for example where different pulses are coupled and no longer independent, or interstitial diffusion mechanisms are present.

\section{ACKNOWLEDGMENTS}

The authors would like to thank all imec colleagues who contributed to this research and the fund for scientific research in Flanders, FWO (Fonds voor Wetenschappelijk Onderzoek, www.fwo.be) who funded this research (grant number SB1S21616N).

${ }^{1}$ S. Beyne, K. Croes, I. De Wolf, and Z. Tőkei, Journal of Applied Physics 119 (2016), 10.1063/1.4947582.

${ }^{2}$ S. Beyne, O. Varela Pedreira, I. De Wolf, Z. Tokei, and K. Croes, Semiconductor Science and Technology 34 (2019).

${ }^{3} \mathrm{~S}$. Beyne, "Electromigration mechanisms in scaled interconnects,".

${ }^{4}$ S. Beyne, L. Arnoldi, I. De Wolf, Z. Tökei, and K. Croes, Applied Physics Letters 111 (2017), 10.1063/1.4989898.

${ }^{5}$ D. Fleetwood, S. Beyne, R. Jiang, S. Zhao, P. Wang, S. Bonaldo, M. McCurdy, Z. Tőkei, I. DeWolf, K. Croes, et al., Applied Physics Letters 114, 203501 (2019)

${ }^{6}$ P. Dutta, P. Dimon, and P. M. Horn, Physical Review Letters 43, 646 (1979).

${ }^{7}$ P. Dutta and P. M. Horn, Reviews of Modern physics 53, 497 (1981).

${ }^{8}$ H. Stoll, Applied Physics A Solids and Surfaces 30, 117 (1983).

${ }^{9} \mathrm{C}$. Heiden, Physical Review 188, 319 (1969)

${ }^{10} \mathrm{M}$. Celasco, F. Fiorillo, and P. Mazzetti, Physical Review Letters 36, 38 (1976).

${ }^{11}$ P. Shewmon, Diffusion in solids (Springer, 2016).

${ }^{12}$ S. Beyne, K. Croes, M. H. V. D. Veen, O. V. Pedreira, Q. Qi, I. D. Wolf, and Z. Tökei, Interconnect Technology Conference (IITC), 2017 IEEE International (2017). 


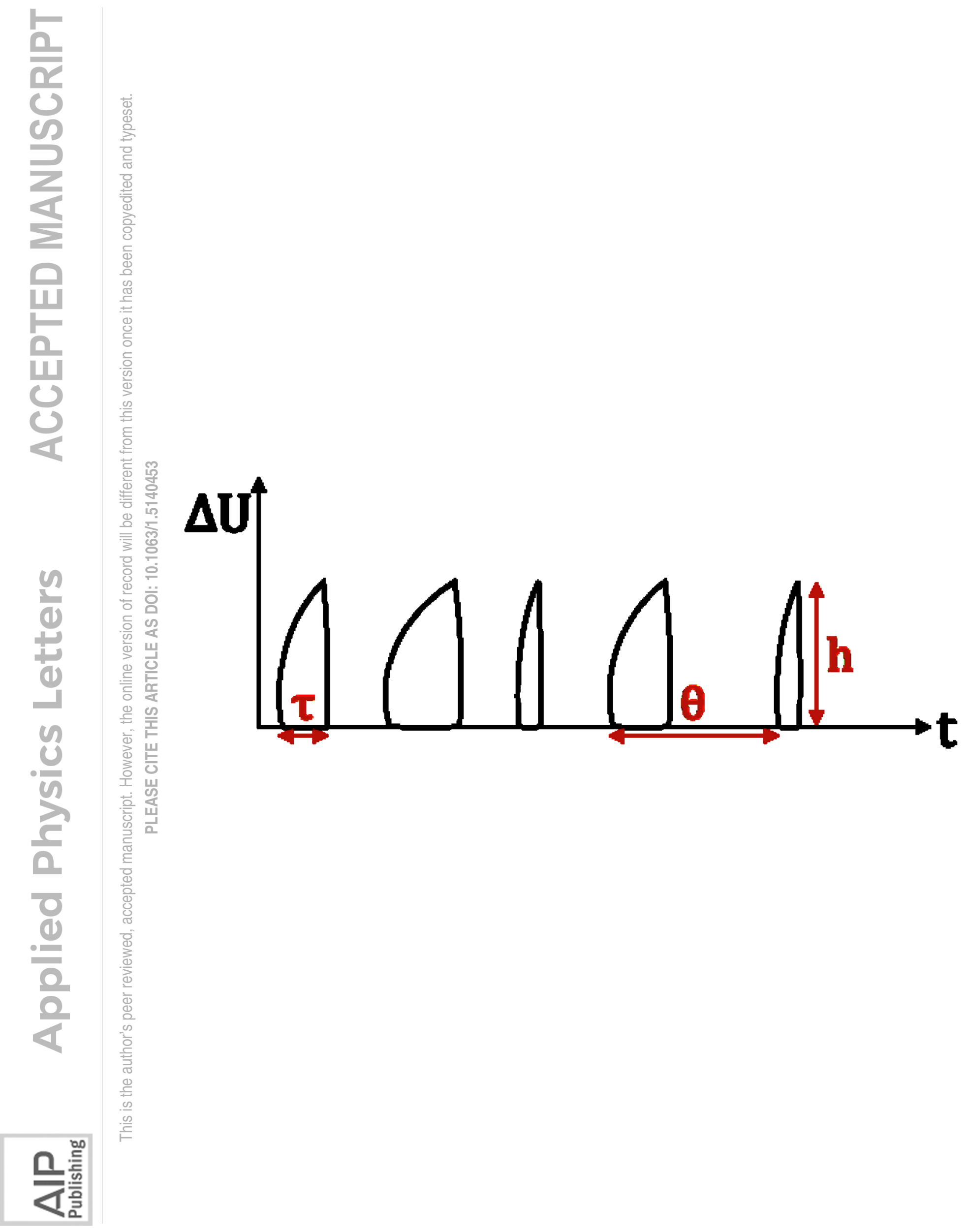




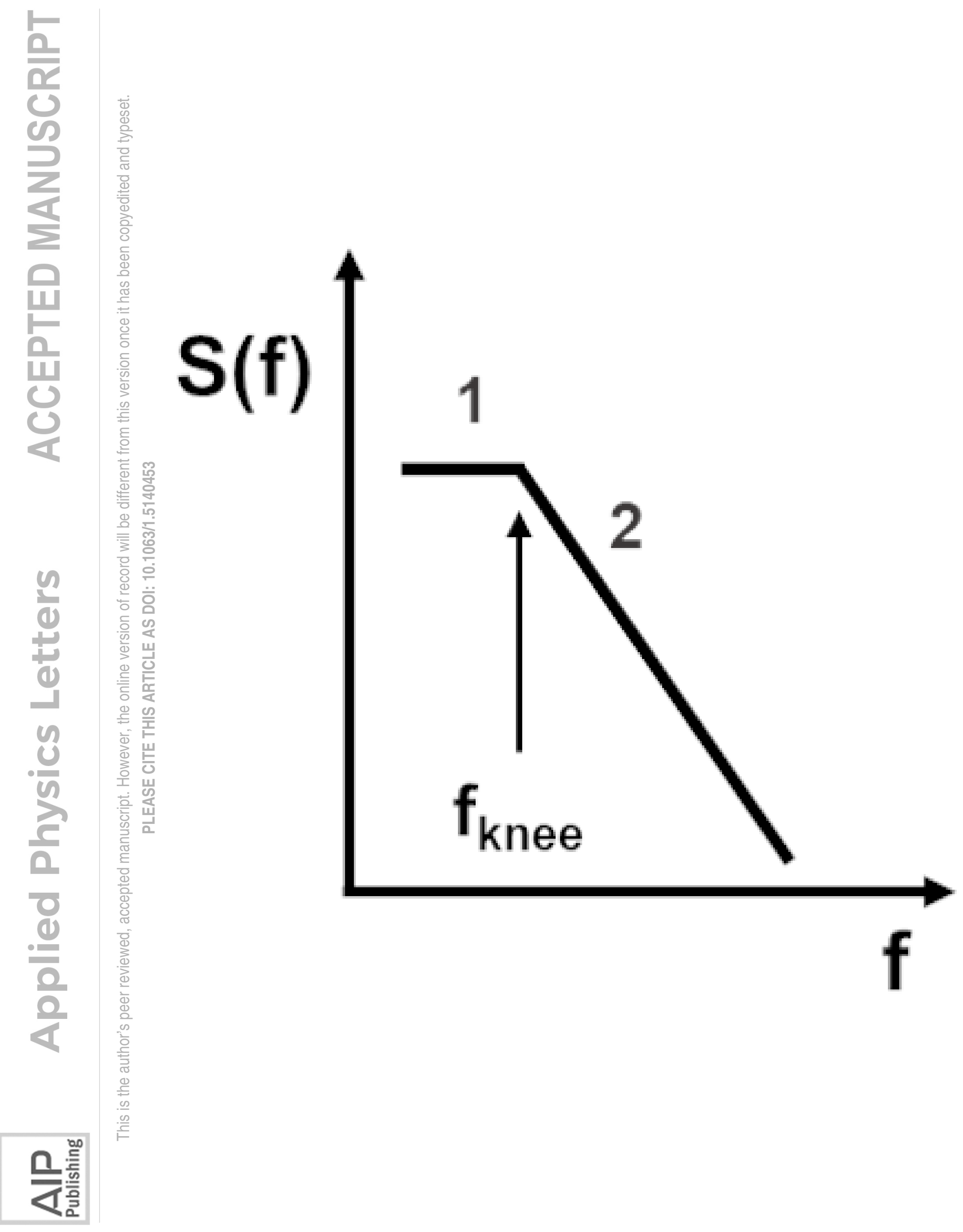




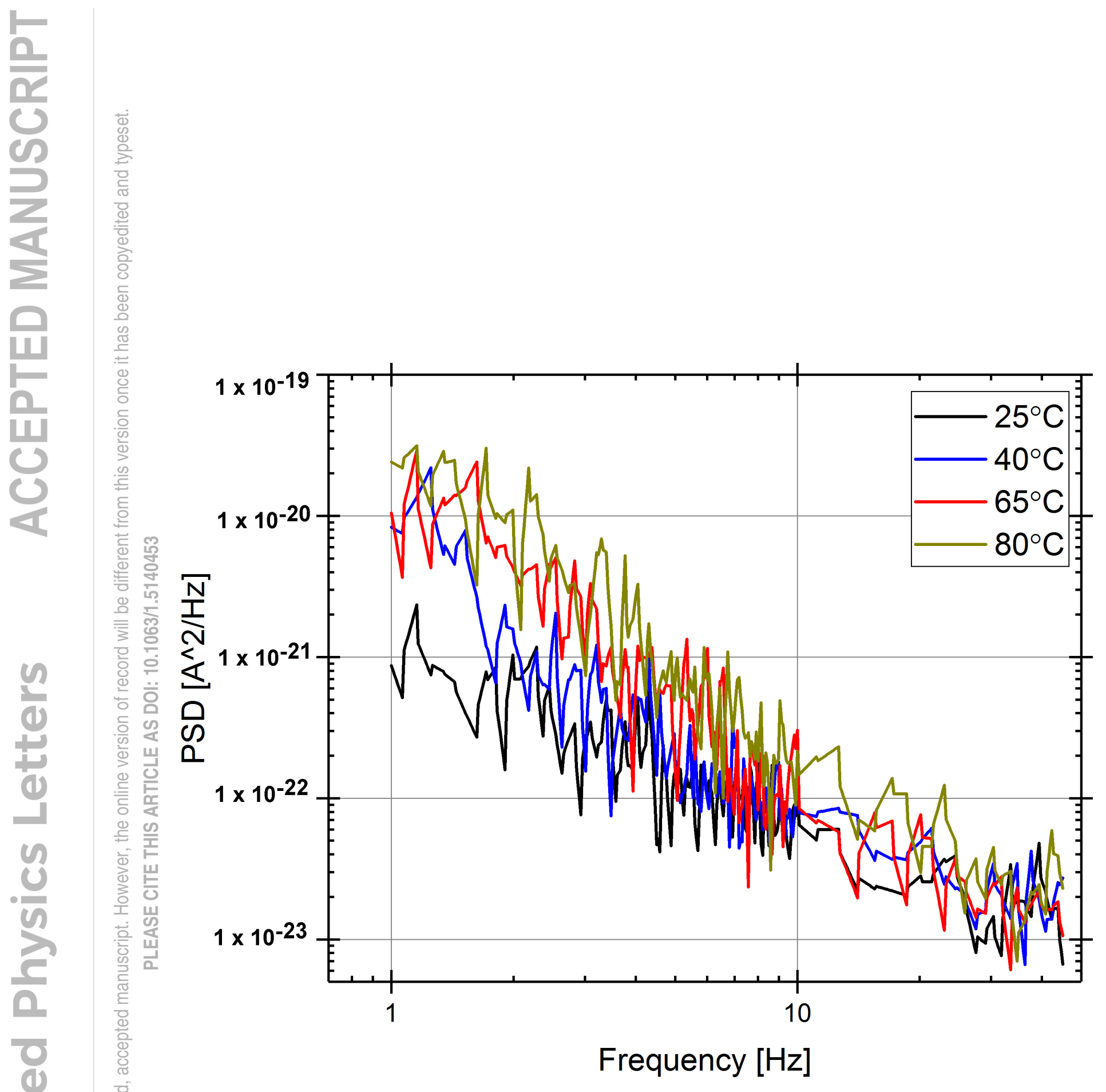

兄耪高 
Temperature $\left[{ }^{\circ} \mathrm{C}\right]$

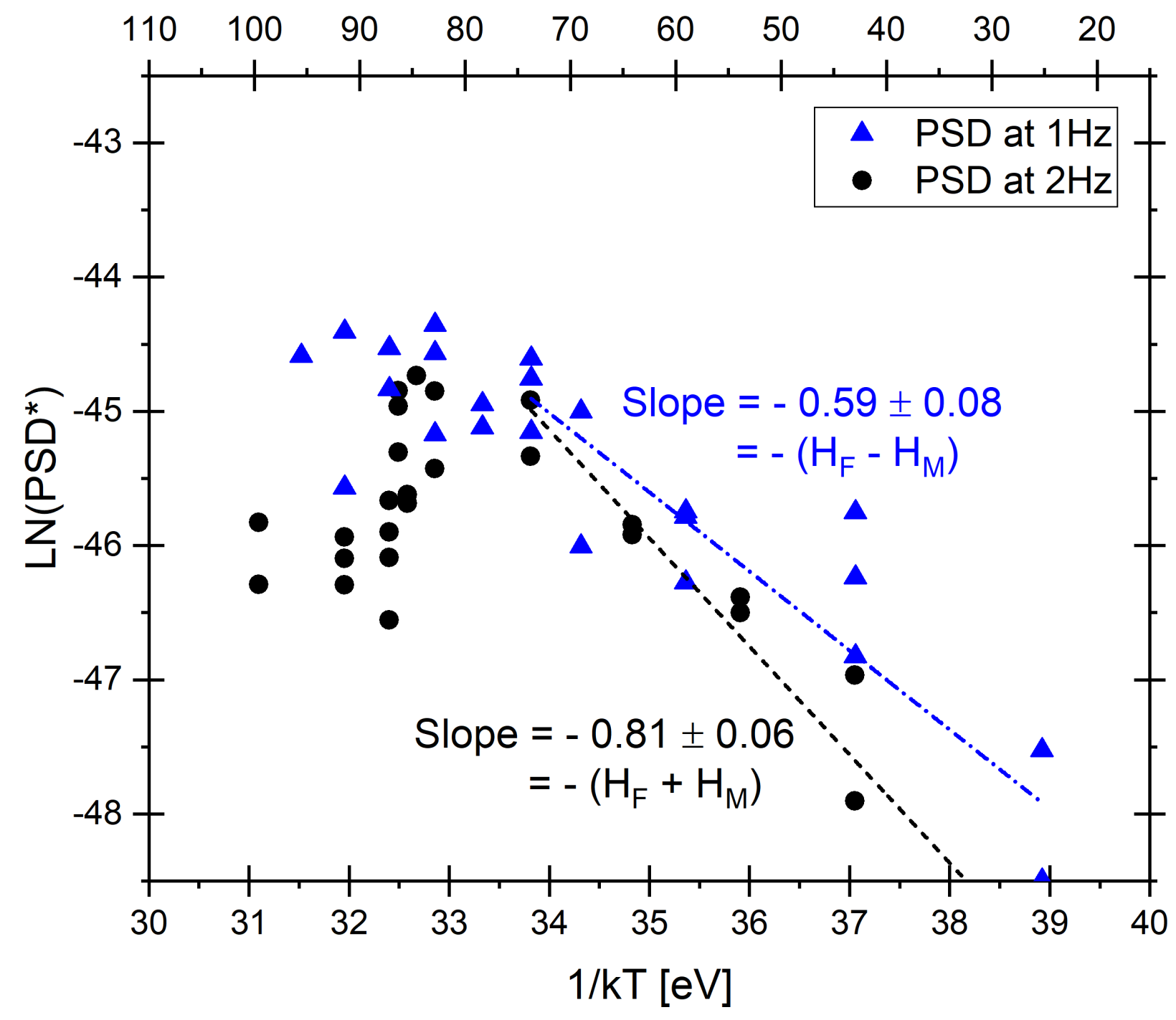




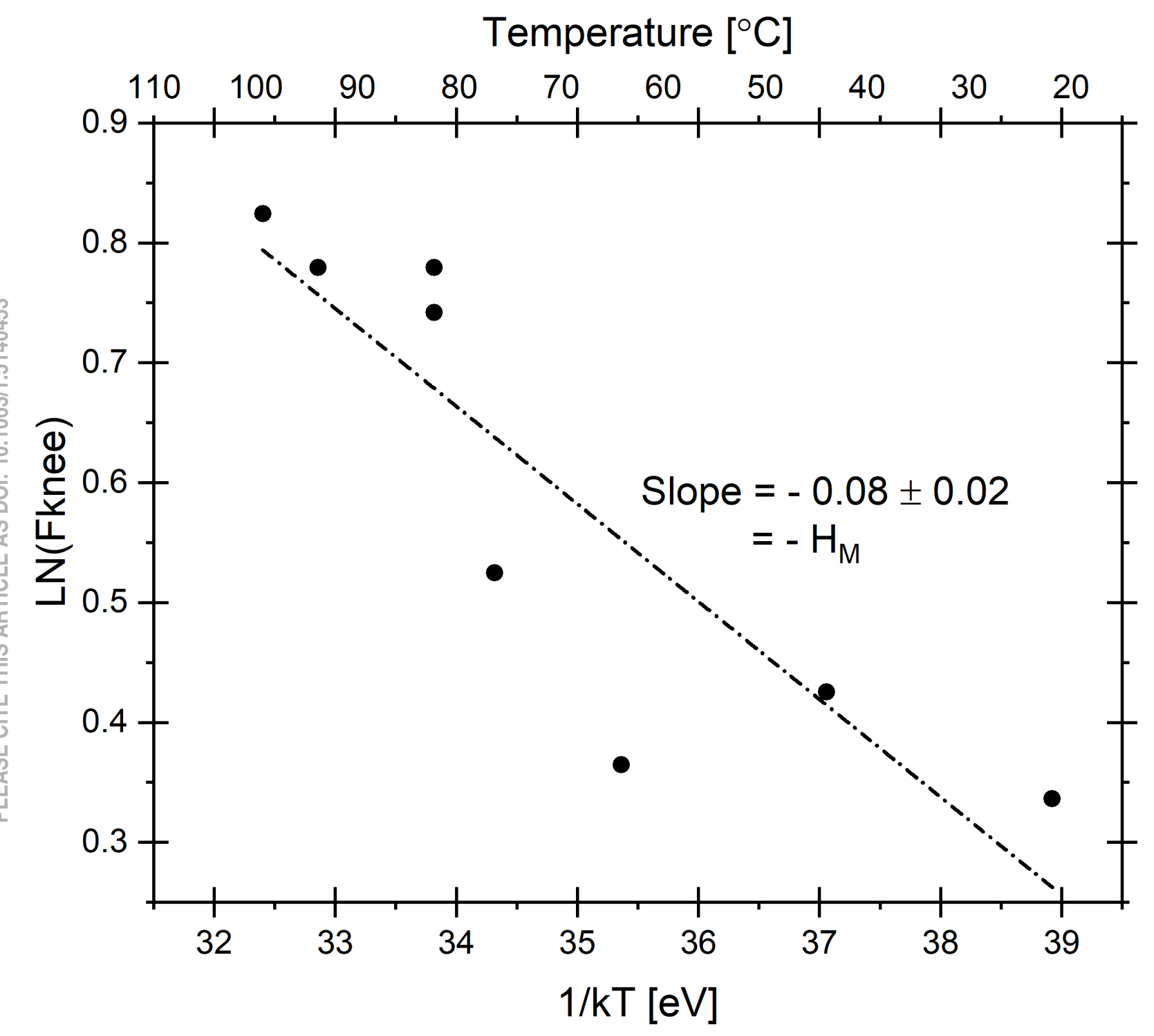


(a)

Temperature $\left[{ }^{\circ} \mathrm{C}\right]$

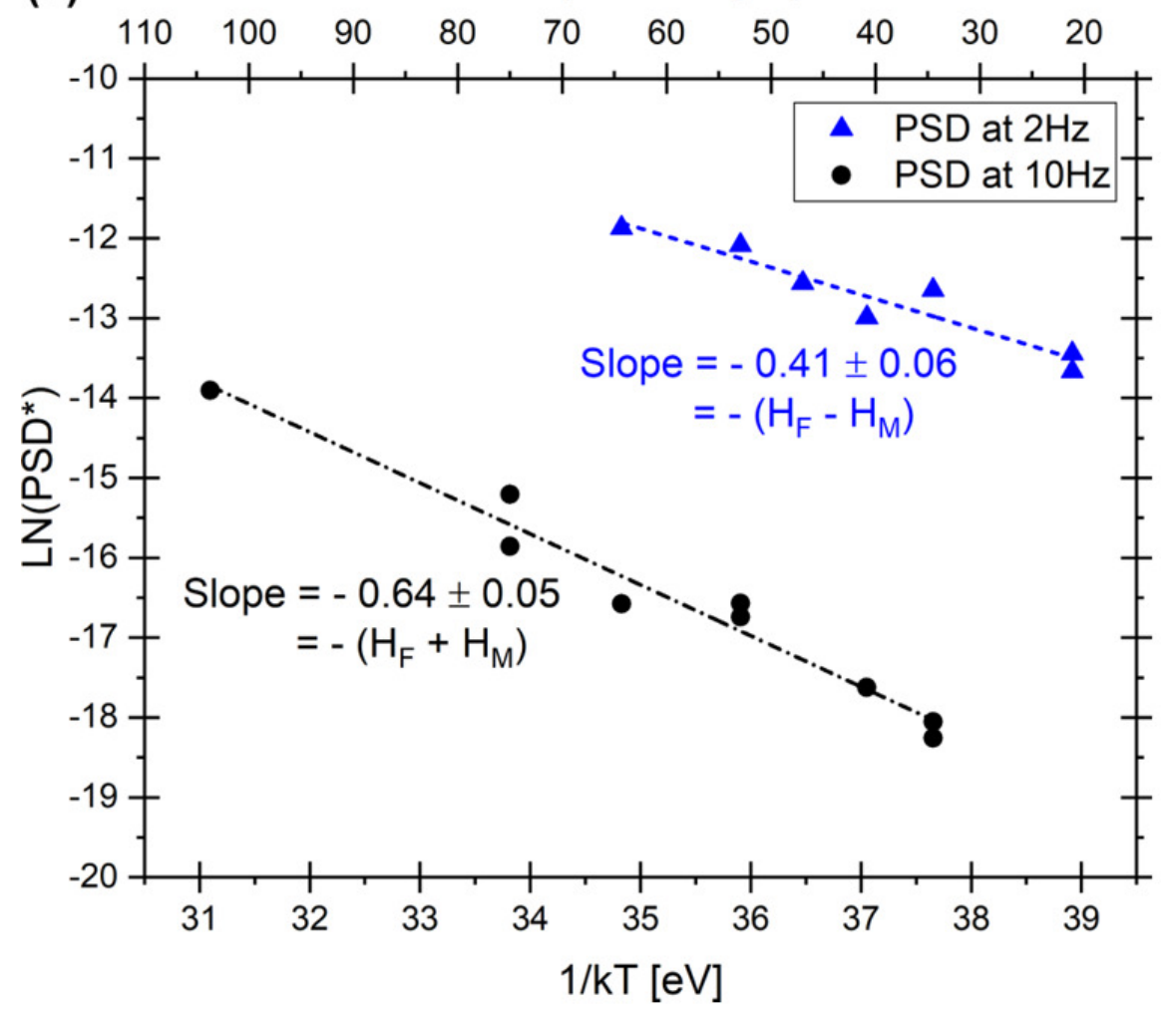

(b)

Temperature $\left[{ }^{\circ} \mathrm{C}\right]$

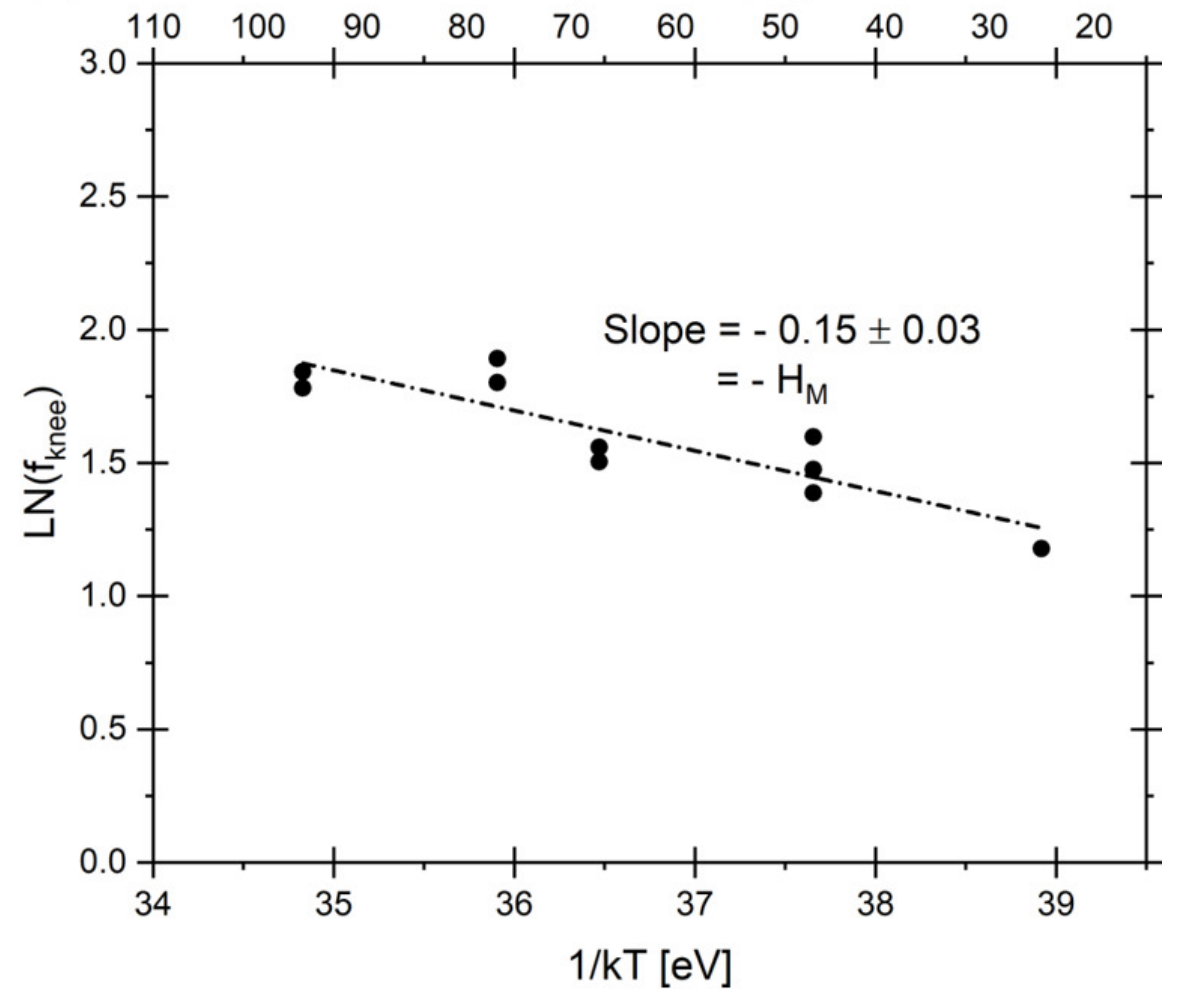

